\title{
The Willingness of Undergraduate Nursing Students Regarding Utilizing CAM as a Preventative Method of Coronaviruses
}

\author{
Najla A. Barnawi ${ }^{* 1,2}$, Hind S. Al-Ghadeer ${ }^{1,2}$, Madhawi M. BinSaran ${ }^{1}$, Walaa, S. Bin-Muneef ${ }^{1}$, \\ Maryam S. Alroilai ${ }^{1}$, Shahad M. Alahmed ${ }^{1}$ \\ ${ }^{1}$ College of Nursing, KSAU-HS, Riyadh 14611, Saudi Arabia \\ ${ }^{2}$ King Abdullah International Medical Research Centre (KAIMRC), Ministry of National Guard Health Affairs, \\ Riyadh, Saudi Arabia
}

"Corresponding author: Najla A. Barnawi; barnawin@ksau-hs.edu.sa

Received 21 February 2021;

Accepted 26 March 2021;

Published 01 April 2021

\begin{abstract}
In response to the global awareness about the Coronavirus, mainly COVID-19, developing an educational-video tool regarding the utilization of Complementary and Alternative Medicine (CAM) as a preventative method of Coronaviruses. It is an ideal resource for nursing students, who are a cornerstone of the continuity of care and partnerships with high-risk individuals, families, and communities. This study examines the impact of the CAM video session on the level of attitude among Saudi undergraduate female nursing students in Riyadh, Saudi Arabia. A prepost tests quasi-experimental study was conducted among 174 students to examine their attitude changes before and after they exposed the CAM educational video. The results suggesting that the CAM video session significantly improves students' attitudes $(\mathrm{M}($ differences $)=55.2068, \mathrm{SD}=$ 5.9964, $[\mathrm{t}(174)=121.444, \mathrm{p}<.001])$; Further, the number of utilizing the CAM therapy mainly the herbal methods was increased after the exposure to CAM video (pre-test $n=76,43.7 \%$ vs. post-test $n=130,74.7 \%$ ). In conclusion enhancing the students' attitudes toward utilizing CAM therapy as preventive measures is a core aspect of public health. Further, implementing such attractive, simple, and evidence-based tools increases the utilization of accessible and available CAM resources such as herbal remedies. Accordingly, there is a need to examine the CAM video tool at a larger scale and not merely focus on the healthcare professionals but should include population-based scope.
\end{abstract}

Keywords: Attitude, Complementary-Alternative Medicine, COVID-19, Nursing Students, Utilization

\section{Introduction}

Traditions and beliefs are fundamental elements in sustaining the consistency of society; one of these traditions is the practice of Complementary and Alternative Medicine (CAM). Using CAM mainly in the Middle East is one of the favourable customs of managing various types of diseases, mainly among the Saudi population. Based on bibliometric analysis published in 2015, Saudi Arabia (SA) exceeds the ranking of scientific research in integrative and complementary medicine (ICM) among Arab countries by $25 \%{ }^{[1]}$. Further, more than $50 \%$ of the Saudi population who use CAM live in Riyadh, the capital city ${ }^{[2,3]}$.

The practice of CAM in SA has a religious-base, as it is a part of the Holy Quran therapy such as using honey, mushrooms, black seed, myrrh, or prophetic medicine such as Alhijama or cupping medicine ${ }^{[3-7]}$. In addition to the religious-base, the Saudi healthcare Vision 2030 recommends that healthcare practitioners enhance integrating all the socio-cultural factors that promote the quality of life among the Saudi population, such as CAM ${ }^{[8]}$. During 2008, the Ministry of Health (MOH) established the centre for complementary and alternative medicine to regulate CAM practices within the healthcare services ${ }^{[4,7]}$.

Therefore, and in response to the current global COVID-19 precautions, studying CAM is required in the Saudi context, mainly among nursing students. Targeting nursing students is ideal for exemplifying the foundation of holistic care as they are the 'cornerstones' of their practice model, including continuity of care and partnership with infectious diseases. Thus, the nurses may initiate the first step of establishing competent skills in preventing illnesses.

Despite the utilizing CAM as a culturally based practice in SA, national evidence indicates its effectiveness in managing various chronic diseases symptoms as safe and a beneficial intervention that overcomes the Medicalized-based treatment 
demands ${ }^{[9-14]}$. Others examined the satisfaction of utilizing CAM in managing different types of pain. For instance, Albedah et al. (2015) claimed that cupping therapy reducing back pain among $80 \%$ of the participants with a significant level $(\mathrm{p}=0.0001)^{[15]}$. Further, Aldahash et al. (2012) claimed $72 \%$ out of 399 participants reported that CAM reduced their abdominal pain and headaches at a significance level of $\mathrm{p} 0.05^{[12]}$. CAM has a positive impact on managing psychiatric disorders as well and that includes depression, anxiety, and reduced mental alertness ${ }^{[12,15-17]}$

Internationally, some scholars examined the role of CAM in managing infection diseases, considering the resistance of medical antibiotics and antibodies products ${ }^{[18]}$. Despite the limited national clinical trials in such topic, there are several international studies recommend utilizing CAM to manage respiratory tract infections ${ }^{[19]}$. Evidence indicates that Caihu, Shuanghuanglian, Yuxincao and Qinkailing injections had a positive effect in managing and treating several respiratory virial or bacterial infections ${ }^{[20]}$. Sfeir et al. 2013 claimed that Cinnamomum verum, Cymbopogon citratus, Thymus vulgaris CT thymol, Origanum compactum, and Satureja montana essential oils exhibited significant antibacterial activity mainly against streptococcus pyogenes ${ }^{[21]}$. According to Hokari et al., 2012 orally administering Shahakusan decreased infiltration of inflammatory cells in the bronchoalveolar spaces and damage of desquamated mucosal epithelia of bronchiole and exerts antiviral effect in mice by its immunomodulating activity through action of NK cells and antiinflammatory activity in the lung ${ }^{[22]}$.

It is essential to investigate the utilization of CAM as a preventative method from Corona diseases, mainly among Saudi nursing students. Assessing their attitudes regarding the CAM and its impact on preventing the Corona disease is a core in current time. Hye Hwang et al., 2020 indicated that $76.1 \%$ out of 331 South Korean patients used one or more types of CAM modalities during MERS outbreak, where the mass media was the main source of information. Establishing a media platform is of paramount importance to provide reliable information and ensure the safety of its use ${ }^{[23]}$. The current Saudi healthcare system is in a transformation process and considering adding CAM in healthcare [7]. Regarding such a socio-cultural perspective, sustaining the concept of holistic care within the nursing profession is crucial.

Therefore, integrating and examining the attitude of utilizing CAM as a preventative approach of various infectious diseases such as Coronavirus mainly among the young generation of university students ${ }^{[24]}$. There are several studies, highlighted the importance of assessing attitude among undergraduate students, mainly in using the CAM in various healthcare context as well as the academic organizations ${ }^{[17,24,25]}$. However, no study was conducted in Saudi nursing students regarding such topic. It has been shown that CAM is one of the highly integrated and sociocultural constructed phenomena ${ }^{[26]}$. Therefore, assessing and exploring the level of attitude provides more in-depth insights into the clinical and cultural aspects of CAM and one of the dangerous infectious diseases such as Corona, which is essential to maintain holistic care ${ }^{[27]}$.

This study aims to examine the impact of CAM video sessions on the level of attitude among Saudi undergraduate female nursing students at King Saud Bin Abdulaziz University (KSAUHS) in Riyadh. Examine the impact of the CAM video module on the number of utilizing different types of CAM during the COVID19 pandemic. Further, identify the existence of a relationship between the students' attitudes and their demographical data on the utilization of CAM modalities.

\section{Materials and Methods}

This study conducted at the College of Nursing-Riyadh (CON-R), which is affiliated to King Saud bin Abdulaziz University for Health Sciences (KSAU-HS). The study has an online video-based module that accessible electronically via a link. The video integrated into a streamlined system, which is a third-party multimedia creation that hosts the participants all in one place.

During the Spring 2020 semester, each participant who was willing to participate in the study and met the study inclusion and exclusion criteria immediately accessed the CAM module through their (Whatsapp) accounts. The participants received oral and written instructions about the aim of the study, how to download, access, and utilize the learning materials of the CAM-video module. All the participants had to finish the self-administered pretest questions, and the self-administered posttest questions at the same period of time. The video contents includes four clips, with specific conetnt in each one of them aiming to enhance the participants positive attitudes towards using CAM during COVID19.

\section{Study Design, Subjects and Sampling}

The study is a quasi-experimental quantitative approach with a pretest-posttest design, which focuses on Saudi undergraduate nursing students who are enrolled as full-time students in one of the accredited national Bachelor's nursing program. The CON-R includes a total number of 400 Saudi undergraduate nursing students at various academic levels. Accordingly, all the participants had to read and speak English and were willing to participate in the study and have basic computer skills to download and navigate the CAM module. The calculated sample size out of 300 nursing students is 147 nursing students, with a confidence interval of $95 \%$, a $5 \%$ margin of error, and a $50 \%$ population proportion. A convenience sampling technique was used to recruit the study participants.

\section{Data Collection \& Instruments}

The demographic and previous knowledge and experience of CAM information was covered by a set of questions eight questions.It includes the participants' age, academic level, the region of living. Additionally, the participants will be asked for some specific demographic data, such as if they had any previous knowledge or utilization of CAM and to verify the source(s) of their information. While the participant's attitud level toward CAM utilization as a preventative methods of COVID-19 was covered by the CAM Health Belief Questionnaire (CHBQ), which has a seven-point Likert rating scale that ranges from (7-49) where 7 indicates the lowest attitude level and 49 is the highest level. The tool of CHBQ is a validated tool with strong internal consistency as its Cronbach's coefficient is 0.75 .

\section{Data Management and Analysis Plan}

IBM SPSS (C) version 26 (IBM Corporation, 2017) was used to analyze descriptive and inferential statistics for this study. Descriptive statistics of several demographic factors and factors related to previous experiences or knowledge of CAM was collected for comparison purposes. Furthermore, a independent ttest was used to measure the mean differences in the level of attitude before and after exposure to the CAM video module. Furthermore, it compares the mean differences against the study hypotheses at a $95 \%$ confidence interval to determine if the differences between these pretest and posttest values are statistically significant. The inferential statistics include calculating the $r$ values to examine the correlation between the student's 
attitudes and other variables associated with utilization of CAM during COVID-19.

\section{Results and Discussion}

\section{Participants General Demographic Data}

A total of 172 female undergraduate students participated in this study, where the youth group represented the largest group (50\%), followed by the middle youth group (44.2\%) and late youth group $(5.8 \%)$. Single status represents the largest portion of the study sample $(95.3 \%)$ followed by married $(3.5 \%)$ and divorced status (1.2\%). Participants who live in Eastern region of Riyadh has the highest portion (36\%) followed by the Centre region of Riyadh $(27.9 \%)$. Participants in advanced academic level represented the most considerable portion of the study sample (46.5\%), followed by those who are in the middle academic level (33.7\%). Only $19.8 \%$ represents students in the basic academic levels. $61.6 \%$ of the participants reported that they utilize various CAM modalities before the time of the study, which implied that most of the participants have the basic knowledge about the CAM modalities before the COVID-19 pandemic. (Table 1)

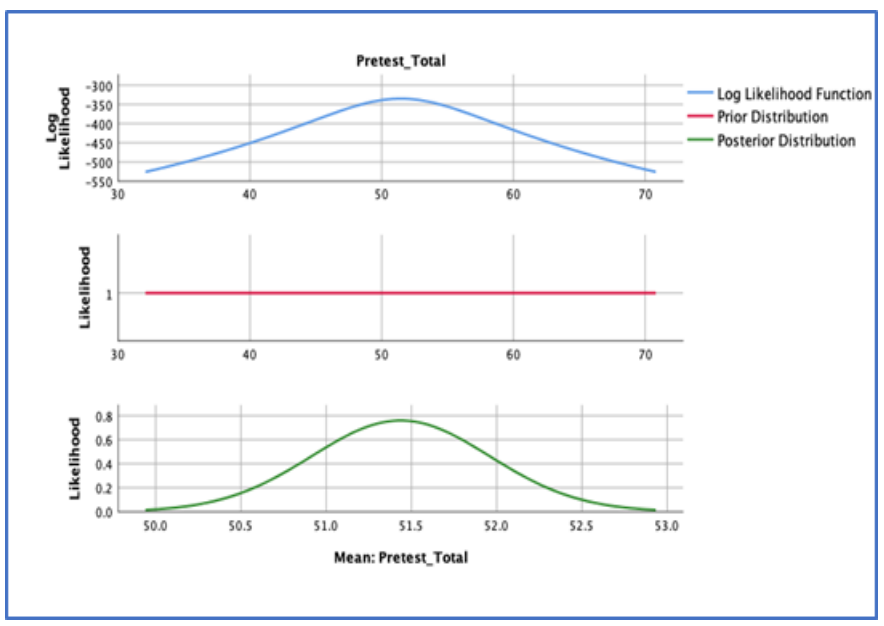

Descriptive Statistics: Students Attitudes of Utilizing CAM During COVID-19

Overall, there is an increase of the post-test mean scores of the student's attitudes (55.2069) compared to the pre-test scores (51.4368) (See Figure 1). The Herbal/ Botanical/ Supplements modality has the highest percentage (pre-test $=44.19$ vs. posttest $=74.42 \%$ ), followed by Meditation/ Yoga/ Relaxation modality (pre-test $=39.54 \%$ vs. post-test $=44.19 \%$ ). However, both the Acupuncture and Ayurveda modalities have the lowest percentage compared to other modalities (post-test $=14 \%$ ) $($ Table 2)

CAM Video Module \& Students Attitudes of Utilizing CAM during COVID-19

Testing the hypothesis indicates that an increase in the mean posttest attitudes scores toward utilization of CAM $(\mathrm{M}=55.21$, $\mathrm{SD}=6.03133)$ compared to the pre-test scores $(\mathrm{M}=51.6163, \mathrm{SD}=$ 6.70893, Table 2). Based on the dependent samples t-test result, the null hypothesis of equal resilience of the attitude means of the pretest and post-test was rejected, $\mathrm{t}(172)=-10.390, \mathrm{p}=0.000$ (Table $3)$. Thus, the mean of the post-test attitude scores of utilizing the CAM was statistically higher than the pre-tests scores mean.

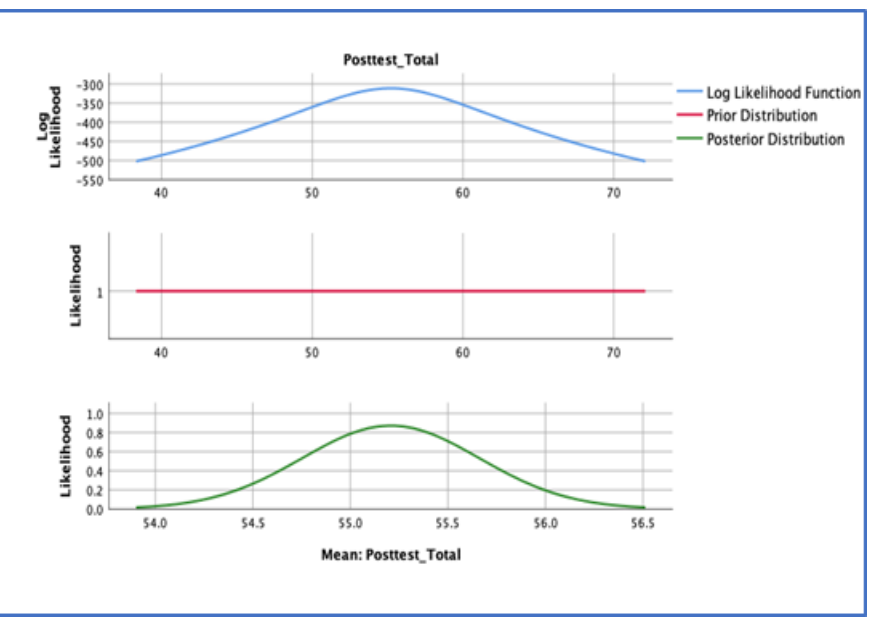

Figure 1: Mean Differences: Pre-test vs. Post-test Utilization of CAM Modalities

Table 1: Participants Demographical Data.

\begin{tabular}{|c|c|c|}
\hline \multicolumn{3}{|l|}{ Age } \\
\hline Age Group & Frequency & Percent \\
\hline Early Youth (18-20) & 86 & 50.0 \\
\hline Middle Youth (21-23) & 76 & 44.2 \\
\hline Late Youth (23-25) & 10 & 5.8 \\
\hline \multicolumn{3}{|l|}{ Relationship Status } \\
\hline Status & Frequency & Percent \\
\hline Married & 6 & 3.5 \\
\hline Divorced & 2 & 1.2 \\
\hline Single & 164 & 95.3 \\
\hline \multicolumn{3}{|l|}{ Region of Living } \\
\hline Region in Riyadh & Frequency & Percent \\
\hline Centre & 48 & 27.9 \\
\hline Southern & 26 & 15.1 \\
\hline Northern & 14 & 8.1 \\
\hline Western & 22 & 12.8 \\
\hline Eastern & 62 & 36.0 \\
\hline \multicolumn{3}{|c|}{ Academic Educational Level } \\
\hline Academic Level & Frequency & Percent \\
\hline Basic & 34 & 19.8 \\
\hline Middle & 58 & 33.7 \\
\hline Advanced & 80 & 46.5 \\
\hline
\end{tabular}




\begin{tabular}{|c|c|c|}
\hline \multicolumn{3}{|c|}{ Previous use of complementary and alternative medicine } \\
\hline CAM Previous Status & Frequency & Percent \\
\hline Yes & 106 & 61.6 \\
\hline No & 66 & 38.4 \\
\hline
\end{tabular}

Table 2: Descriptive Statistics CAM Module on Utilization of CAM Modalities.

\begin{tabular}{|l|l|l|l|l|}
\hline Type of CAM & Pre-test & Post-test \\
\hline & Frequency & Percent & Frequency & Percent \\
\cline { 2 - 5 } Acupuncture & 4 & 2.33 & 24 & 14 \\
Herbal/ Botanical/ Supplements & 76 & 44.19 & 128 & 74.42 \\
Utilization of Massage & 70 & 40.7 & 74 & 43.023 \\
Spirituality/ Prayer & 42 & 24.42 & 58 & 33.72 \\
Meditation/ Yoga/ Relaxation & 68 & 39.54 & 76 & 44.19 \\
Homeopathy & 4 & 2.33 & 38 & 22.1 \\
Ayurveda & 0 & 0 & 24 & 14 \\
\hline
\end{tabular}

Table 3: CAM Video Module (Pre-test \& Post-test Attitude Scale Scores).

\begin{tabular}{|l|l|l|l|l|}
\hline Paired Samples Statistics & Mean & N & Std. Deviation & Std. Error Mean \\
\cline { 2 - 5 } Pre-test Attitude Total & 51.4368 & 172 & 6.87578 & .52125 \\
Post-test Attitude Total & 55.2069 & 172 & 5.99641 & .45459 \\
\hline
\end{tabular}

Table 4: CAM Video Module (Pre-test \& Post-test Attitude Scale Scores).

\begin{tabular}{|c|c|c|c|c|c|c|c|c|}
\hline \multicolumn{9}{|l|}{ Paired Samples Test } \\
\hline & \multicolumn{5}{|c|}{ Paired Differences } & $\mathrm{t}$ & $\mathrm{df}$ & Sig. (2-tailed) \\
\hline & \multirow[t]{2}{*}{ Mean } & \multirow[t]{2}{*}{ Std. Deviation } & \multirow[t]{2}{*}{$\begin{array}{l}\text { Std. Error } \\
\text { Mean }\end{array}$} & \multicolumn{2}{|c|}{$\begin{array}{l}95 \% \text { Confidence Interval } \\
\text { of the Difference }\end{array}$} & & & \\
\hline & & & & Lower & Upper & & & \\
\hline $\begin{array}{l}\text { Pre-test \& Post-test } \\
\text { Attitude Total }\end{array}$ & -3.77011 & 4.80053 & .36393 & -4.48842 & -3.05181 & -10.360 & 173 & .000 \\
\hline
\end{tabular}

\section{Discussion}

Assessing the nursing students' attitudes regarding the utilization of CAM modalities is essential element in this study considering the current gap in the Saudi literature in this regard ${ }^{[24]}$. However, the study results indicated that there is an increase in the student positive attitude of utilizing CAM mainly the herbs after delivering the study intervention. Based on Alzahrani et al (2016), 40 percent out of 242 Saudi medical students reported that herbs modality is the best, which is combatable with the current study result. Enhancing the nursing students' attitudes toward the utilization of CAM modalities mainly herbs sustains the concept of providing holistic-care toward their patients mainly during COVID-19 pandemic.

The study result indicates that 74.42 percent of the students had positive attitude toward utilizing CAM as a preventative method of COVID-19 after watching the CAM video model. Such results of positive attitudes of utilizing CAM were found among general physicians, nurses, medical and nursing students ${ }^{[28-39]}$. However, in a study that compared medical and nursing students, it was found that nursing students had more positive attitudes than medical students toward complementary and alternative medicine therapies ${ }^{[30,31]}$.

According to the other studies findings, there is a lack of evidence, training, and appropriate equipment, which are barriers of utilizing the CAM therapies mainly the herbs modality $[30,34,35,37,40]$. Consequently, students may perceive CAM modality is not very effective in the clinical or long-term life span because of their personal beliefs and attitudes regarding these barriers. It is highly important to integrate such CAM-video educational-based model within nursing curricula to sustain positive attitude of utilizing CAM among nursing students.

The lack of relevant literature focused on utilizing CAM within the Saudi nursing healthcare context was one of the key challenges in this study. Therefore, there is a need to conduct highly advanced types of studies such as randomized control trails (RCT) that aim to examine the impact of this CAM video educational-based module on providing competent care by the healthcare professionals. Further, mixed methods and qualitative research studies is highly recommended to assess the level of satisfactions of nursing students when providing care utilizing the CAM-based knowledge. Conducting such types of studies develops a robust scientific level of evidence that concern clinical and cultural aspects to manage the current pandemic especially considering the instability of the COVID-19 pandemic circumstances globally.

There are several limitations in this study; for instance, the study participants were selected through purposive sampling, and the sample was small, and representing only one nursing college and from a single university. The sample consisted entirely of women, so gender differences could not be examined. Therefore, the findings of the present study cannot be generalized. Furthermore, given that attitudes toward CAM are culturally and socially constructed, which were not examined and require further investigation. Future studies should include a large sample. In addition, qualitative studies may provide valuable data regarding attitudes and understanding toward the utilizing CAM as a preventative method of COVID-19.

\section{Conclusions}


Utilizing the CAM modalities is one of the sophisticated socially constructed phenomena, which has tight traditional roots and overlapping cultural ideologies developed throughout history. In response to the current global COVID-19 precautions, studying CAM is required in the various healthcare context, mainly among nursing students. Targeting nursing students is ideal for exemplifying the foundation of holistic care. A pretest-posttest one group quantitative approach was the main research design for this study. Regardless the limitations of this design and the study in general, the result indicated that there was a statistically significant positive impact of the CAM video educational-based module on the students' attitude regarding utilizing CAM as a preventative method against COVID-19. This implied that the module is a recommended educational tool that helps the nurses to optimize and provide culturally and clinically competent nursing care. Further, implementing such attractive, simple, and evidence-based tools increases the utilization of accessible and available CAM resources such as herbal remedies.

\section{Ethics approval and consent to participate}

Approval of this study was obtained from the Research unit at CON-R and the Institutional Review Board (IRB) of Human Subject Review Research Office (HSRRC) at King Abdullah International Medical Centre (KAIMRC) at KSAU-HS with (RYD20-419812-22123) on June 10th, 2020. The participants received an electronic consent form through the management system of the KSAU-HS, which has a short description of the study and asks likely subjects about their willingness to participate in the study. They were asked to electronically sign the consent form, a requirement for them to access the pre-test, CAM-Video module, and the post-test content. Their participation in this study was entirely voluntary, and they were free to discontinue the completion of the study at any time without penalty. No personal identifiers were collected or reported, and findings were reported as aggregate data. Risks in participating in this study are considered to be minimal. An agreement between the (PI) of this study and the CON-R faculty members who agreed to include the CAM module in their course outlines that the potential subject participation was considered extra-curriculum professional development and would not affect their course grade. All the data saved in one computer, which required password access. In terms of human or animal tissue utilization was not applicable in this study.

\section{List of abbreviations}

CAM: Complementary Alternative Medicine

SA: Saudi Arabia

ICM: Integrative and Complementary Medicine

MOH: Ministry of Health

KSAU-HS: King Saud Bin Abdulaziz University for Health

Sciences

CON-R: College of Nursing-Riyadh

CHBQ: CAM Health Belief Questionnaire

IRB: Institutional Review Board

HSRRC: Human Subject Review Research Office

KAIMRC: King Abdullah International Medical Centre

PI: Principal Investigator

\section{Data Availability}

The readers can access the data underlying the findings of the study, giving links to online repositories and providing deposition codes where applicable based on the journal rules and regulations.

\section{Conflicts of Interest}

The author(s) declare(s) that there is no conflict of interest regarding the publication of this paper.

\section{Funding Statement}

The publication fee of this article is funded by KAIMRC.

\section{Authors' contributions}

NB supervised, analysed, and interpreted the participants' data regarding this study was a major contributor in writing the manuscript. HA provided logistical support throughout the research project. MR developed the content of the Myrrha's video-materials and developed the demographical part of the instrument. SH developed the content of Honey video-materials and translated the first part of the study tool. WM developed the content of Mastic video-materials and collected the study data. All the authors have critically reviewed and approved the final draft and are responsible for the manuscript's content and similarity index. All authors read and approved the final manuscript.

\section{Acknowledgments}

This research was supported by the King Abdullah International Medical Research Center and the College of Nursing Research Center. We would also like to show our gratitude to KSAU-HS authoritative people to facilitate the process of this study and special thanks to the nursing students for sharing their pearls of wisdom with us during this research.

\section{Supplementary Materials}

The contents of the CAM-Video clips include the following

- Clip 1: (Myrrh as a preventative measure against infectious diseases), this clip covers Backgrounds of myrrh utilizations as preventative method from infectious diseases. Evidence of the Myrrha effects and its effects regarding the drug-resistant ones. The impacts of myrrh on stimulating the immune system and white blood cells.

- Clip 2: (Honey as a preventative measure against infectious diseases), this clip covers the socio-cultural and the religious based of utilizing Honey and its benefit. Chemical composition of Honey and its physiological mechanism. Evidence of the honey effectiveness in manging the upper respiratory infections.

- Clip 3: (Mastic resin tree as a preventative measure against infectious diseases), this clip covers the ancient history of mastic uses as a preventative measure for various diseases. Chemical components of mastic, and its effect on enhancing the immune systems. Saudi traditions of utilizing mastic as a preventative method from infectious diseases.

- Clip 4: (Homemade facial masks and their maintenance during COVID-19), this clip covers general guideline about the appropriate utilization, selection of masks during the COVID-19 pandemic and 
the WHO recommendations in making fabric facial masks.

\section{References}

[1] Zyoud, S. H., Zyoud, S. H., Al-Jabi, S. W., Sweileh, W. M. \& Awang, R. Contribution of Arab countries to pharmaceutical wastewater literature: A bibliometric and comparative analysis of research output. Ann. Occup. Environ. Med. (2016) doi:10.1186/s40557-016-0117-0.

[2] AlGhamdi, K. M. et al. Use of complementary and alternative medicine among dermatology outpatients: Results from a national survey. Journal of Cutaneous Medicine and Surgery (2015) doi:10.1177/1203475415584867.

[3] Alrowais, N. A. \& Alyousefi, N. A. The prevalence extent of Complementary and Alternative Medicine (CAM) use among Saudis. Saudi Pharmaceutical Journal (2017) doi:10.1016/j.jsps.2016.09.009.

[4] Al-Bedah, A. et al. Current Status of Traditional and Complementary Medicine Use in Qassim Province, Saudi Arabia. J. Complement. Altern. Med. Res. (2017) doi:10.9734/jocamr/2017/36711.

[5] Elsayed, E. A., El Enshasy, H., Wadaan, M. A. M. \& Aziz, R. Mushrooms: A potential natural source of antiinflammatory compounds for medical applications. Mediators of Inflammation (2014) doi:10.1155/2014/805841.

[6] Khalil, M. K. M., Al-Eidi, S., Al-Qaed, M. \& AlSanad, $\mathrm{S}$. The future of integrative health and medicine in Saudi Arabia. Integr. Med. Res. (2018) doi:10.1016/j.imr.2018.06.004.

[7] Khalil, M. K. M., Al-Eidi, S., Al-Qaed, M. \& AlSanad, S. Cupping therapy in Saudi Arabia: from control to integration. Integr. Med. Res. (2018) doi:10.1016/j.imr.2018.05.002.

[8] Yezli, S. \& Khan, A. COVID-19 social distancing in the Kingdom of Saudi Arabia: Bold measures in the face of political, economic, social and religious challenges. Travel Med. Infect. Dis. (2020) doi:10.1016/j.tmaid.2020.101692.

[9] Abdullah Al-Rowais, N. et al. Knowledge and attitudes of primary health care physicians towards complementary and alternative medicine in the Riyadh Region, Saudi Arabia. Forsch. Komplementarmed. (2012) doi: $10.1159 / 000335814$

[10] Al-Zahim, A. et al. Use of alternative medicine by Saudi liver disease patients attending a tertiary care center: Prevalence and attitudes. Saudi J. Gastroenterol. (2013) doi:10.4103/1319-3767.108477.

[11] Al Moamary, M. Unconventional therapy use among asthma patients in a tertiary care center in Riyadh, Saudi Arabia. Ann. Thorac. Med. (2008) doi:10.4103/18171737.39636.

[12] ALDAHASH, F. \& MARWA, A. Attitude towards the use of complementary and alternative medicine by patients in Saudi Arabia. Biomedica (2012).

[13] Alghadir, A. H., Al-Yousef, H. M., Al-Hussany, F., Hasaneen, A. \& Iqbal, Z. A. Beliefs and attitudes of paramedical college staff towards complementary and alternate medicine. African J. Tradit. Complement. Altern. Med. (2016) doi:10.21010/ajtcam.v13i5.22.
[14] Mohammad, Y. et al. Pattern of traditional medicine use by adult Saudi patients with neurological disorders. BMC Complement. Altern. Med. (2015) doi:10.1186/s12906015-0623-6.

[15] AlBedah, A. et al. The use of wet cupping for persistent nonspecific low back pain: Randomized controlled clinical trial. J. Altern. Complement. Med. (2015) doi:10.1089/acm.2015.0065.

[16] Al-Faris, E. A. The pattern of alternative medicine use among patients attending health centres in a military community in riyadh. J. Fam. Community Med. (2000).

[17] Al Mansour, M. A. et al. Medical students' perceptions of complementary and alternative medicine therapies: A pre-and post-exposure survey in Majmaah university, Saudi Arabia. African J. Tradit. Complement. Altern. Med. (2016) doi:10.4314/ajtcam.v13i1.2.

[18] Baker, J. H., Qiu, J. \& Grine, K. Role of Complementary and Alternative Therapies in Infectious Disease. Primary Care - Clinics in Office Practice (2018) doi:10.1016/j.pop.2018.05.009.

[19] Kok, E. T., Jong, M. C., Gravendeel, B., Van Leeuwen, W. B. \& Baars, E. W. Resistance to Antibiotics and Antifungal Medicinal Products: Can Complementary and Alternative Medicine Help Solve the Problem in Common Infection Diseases? The Introduction of a Dutch Research Consortium. Evidence-based Complementary and Alternative Medicine (2015) doi:10.1155/2015/521584.

[20] Wu, T., Bian, Z. X., Abudu, M., Adams, D. \& Ko, S. G. Complementary and alternative medicine for respiratory tract infectious diseases: Prevention and treatments. Evidence-based Complementary and Alternative Medicine (2014) doi:10.1155/2014/913095.

[21] Sfeir, J., Lefrançois, C., Baudoux, D., Derbré, S. \& Licznar, P. In vitro antibacterial activity of essential oils against streptococcus pyogenes. Evidence-based Complement. Altern. Med. (2013) doi:10.1155/2013/269161.

[22] Hokari, R., Nagai, T. \& Yamada, H. In vivo antiinfluenza virus activity of Japanese herbal (Kampo) medicine, 'shahakusan,' and its possible mode of action. Evidence-based Complement. Altern. Med. (2012) doi:10.1155/2012/794970.

[23] Hye Hwang, J. et al. Complementary and alternative medicine use among outpatients during the 2015 mers outbreak in South Korea: A crosssectional study. BMC Complement. Med. Ther. (2020) doi:10.1186/s12906020-02945-0.

[24] Albadr, B. et al. Attitude of Saudi medical students towards complementary and alternative medicine. $J$. Fam. Community Med. (2018) doi:10.4103/jfcm.jfcm_98_17.

[25] Ahmed, S. et al. Medical students' opinion toward the application of complementary and alternative medicine in healthcare. Saudi J. Med. Med. Sci. (2017) doi:10.4103/1658-631x.194255.

[26] Gale, N. The Sociology of Traditional, Complementary and Alternative Medicine. Sociol. Compass (2014) doi:10.1111/soc4.12182.

[27] Hall, H., Leach, M., Brosnan, C. \& Collins, M. Nurses' attitudes towards complementary therapies: A systematic review and meta-synthesis. International Journal of 
Nursing

Studies

(2017)

doi:10.1016/j.ijnurstu.2017.01.008.

[28] Alshaar, I. A. M. S., Ismail, M. F. S., Yousuf, W. A. A. A. \& Salama, R. E. Knowledge, attitudes and practice of general practitioners towards complementary and alternative medicine in Doha, Qatar. East. Mediterr. Heal. J. (2010) doi:10.26719/2010.16.5.522.

[29] Alzahrani, S. H., Bashawri, J., Salawati, E. M. \& Bakarman, M. A. Knowledge and Attitudes towards Complementary and Alternative Medicine among Senior Medical Students in King Abdulaziz University, Saudi Arabia. Evidence-based Complement. Altern. Med. (2016) doi:10.1155/2016/9370721.

[30] Kinchen, E. V. \& Loerzel, V. Nursing Students' Attitudes and Use of Holistic Therapies for Stress Relief. J. Holist. Nurs. (2019) doi:10.1177/0898010118761910.

[31] Complementary and Alternative Therapies in Nursing. Complementary and Alternative Therapies in Nursing (2018). doi:10.1891/9780826144348.

[32] Pirotta, M. V., Cohen, M. M., Kotsirilos, V. \& Farish, S. J. Complementary therapies: Have they become accepted in general practice? Med. J. Aust. (2000) doi:10.5694/j.1326-5377.2000.tb127932.x.

[33] Abbott, R. B. et al. Medical student attitudes toward complementary, alternative and integrative medicine. Evidence-based Complement. Altern. Med. (2011) doi:10.1093/ecam/nep195.

[34] Akan, H. et al. Knowledge and attitudes towards complementary and alternative medicine among medical students in Turkey. BMC Complement. Altern. Med. (2012) doi:10.1186/1472-6882-12-115.
[35] Ameade, E. P. K., Amalba, A., Helegbe, G. K. \& Mohammed, B. S. Medical students' knowledge and attitude towards complementary and alternative medicine - A survey in Ghana. J. Tradit. Complement. Med. (2016) doi:10.1016/j.jtcme.2015.03.004.

[36] Loh, K. P., Ghorab, H., Clarke, E., Conroy, R. \& Barlow, J. Medical students' knowledge, perceptions, and interest in complementary and alternative medicine. J. Altern. Complement. Med. (2013) doi:10.1089/acm.2012.0014.

[37] Samara, A. M., Barabra, E. R., Quzaih, H. N. \& Zyoud, S. H. Use and acceptance of complementary and alternative medicine among medical students: A cross sectional study from Palestine. BMC Complement. Altern. Med. (2019) doi:10.1186/s12906-019-2492-x.

[38] Avino, K. Knowledge, attitudes, and practices of nursing faculty and students related to complementary and alternative medicine: A statewide look. Holist. Nurs. Pract. (2011) doi:10.1097/HNP.0b013e318232c5aa.

[39] Sibiya, M. N., Maharaj, L. \& Bhagwan, R. Perceptions of professional nurses towards complementary and alternative modalities (CAM) in the uMgungundlovu District, KwaZulu-Natal. Int. J. Africa Nurs. Sci. (2017) doi:10.1016/j.ijans.2017.06.001.

[40] Stuber, M., Desylvia, D., Fung, C. C., Bazargan-Hejazi, S. \& Cooper, E. The knowledge, attitudes and usage of complementary and alternative medicine of medical students. Evidence-based Complement. Altern. Med. (2011) doi:10.1093/ecam/nen075. 